\title{
Bone Mineral Content and Density in Rett Syndrome and Their Contributing Factors
}

\author{
AMANDA L. JEFFERSON, HELEN J. WOODHEAD, SUE FYFE, JULIE BRIODY, AMI BEBBINGTON, BOYD J. STRAUSS, \\ PETER JACOBY, AND HELEN LEONARD
}

\begin{abstract}
School of Biomedical Sciences [A.L.J.], School of Public Health [S.F.], Curtin University of Technology, Perth, Western Australia 6845, Australia; Telethon Institute for Child Health Research, Centre for Child Health Research [A.L.J., A.B., P.J., H.L.,], The University of Western Australia, Perth, Western Australia 6872, Australia; Department of Paediatric Endocrinology [H.J.W.] Sydney Children's Hospital, Randwick, New South Wales 2031, Australia; Department of Nuclear Medicine [J.B.], The Children's Hospital at Westmead Sydney, New South Wales 2145, Australia; Body Composition Laboratory [B.J.S.] Monash Medical Centre and Southern Clinical School, Monash University, Clayton, Victoria 3800, Australia
\end{abstract}

\begin{abstract}
This study used densitometry to investigate the areal bone mineral density (aBMD) and bone mineral content (BMC) in an Australian Rett syndrome cohort and to assess how factors such as genotype, epilepsy, BMI, and mobility affect these parameters. The influence of lean tissue mass (LTM) and bone area (BA) on total body BMC (TBBMC) was also investigated. Participants, recruited from the Australian Rett Syndrome Database (ARSD), had TBBMC and lumbar spine (LS) and femoral neck (FN) aBMD measured using Dual energy $\mathrm{x}$-ray absorptiometry. Mean height standardized $Z$ scores and CIs for the bone outcomes were obtained from multiple regression models. The mean height $Z$ score for the FN aBMD was low at -2.20 , while the LS aBMD was -0.72 . The TBBMC mean height $Z$ score was -0.62 , although once adjusted for BA and LTM, the mean was above zero, suggesting that low BMC can be explained by narrow bones and decreased muscle mass, likely secondary to decreased mobility. Multiple linear regression identified the p.R168 $\times$ and p.T158M mutations as the strongest predictors of low $\mathrm{aBMC}$ and BMD for all bone outcomes. The strong relationship between genotype, BMC, and aBMD is likely underpinned by the strong relationship between LTM, mobility, and bone outcome measures. (Pediatr Res 69: 293-298, 2011)
\end{abstract}

$\mathrm{R}$ ett syndrome (RTT) is a progressive neurodevelopmental disorder, with a diagnosis incidence of 1.09 per 10,000 females by the age of $12 \mathrm{y}$ (1). RTT predominantly occurs in females, who can be categorized as having either a typical (classic) or an atypical (variant) form (2). Severity varies, but the motor and cognitive disability is nevertheless severe (2). In 1999, mutations on the X-chromosome in the gene encoding methyl-CpG binding protein $2(M E C P 2)$ were identified as the principal genetic cause of RTT $(3,4)$. Although there are more than 240 types of pathogenic nucleotide changes, eight common missense and nonsense mutations have been identified (5).

In a population-based study, the fracture risk in RTT has been found to be nearly four times the general population rate (6). The presence of epilepsy almost doubled the risk of

Received May 10, 2010; accepted October 27, 2010.

Correspondence: Helen Leonard, M.B.Ch.B., Centre for Child Health Research, The University of Western Australia, Telethon Institute for Child Health Research, Perth, Western Australia 6872, Australia; e-mail: hleonard@ichr.uwa.edu.au

Supported by the National Institute of Health grant 5R01HD043100-05, NHMRC \#303189. H.L. was previously funded by a NHMRC program grant (\#353514). Her current funding is from an NHMRC Senior Research Fellowship \#572568. fracture as did the use of two or more antiepileptic drugs (6). In another investigation, valproate was shown specifically to increase the fracture rate 3-fold (7).

Bone mass has been shown to be a strong predictor of fracture risk in adults (8) and children $(8,9)$. Radiographic and ultrasound studies have demonstrated osteopenia and reduced cortical thickness in RTT (10-12). In addition, using dual energy $\mathrm{x}$-ray absorptiometry (DXA), the areal bone mineral density (aBMD) and bone mineral content (BMC) have been shown to be reduced in studies involving 20 (13) and 50 females with RTT (14).

Multiple factors are thought to influence bone mass and fracture risk. Eighty percent of peak bone mass may be determined by genetic predisposition (15). It is feasible that the reduced bone density seen in RTT may be directly associated with the type of $M E C P 2$ mutation. The ultrastructure and density of bone in mice with and without the Mecp 2 protein have recently been examined (16). Those without the functional protein showed growth retardation, abnormal growth plates with irregularly shaped chondrocytes and decreased cortical, trabecular and calvarial bone (16). Thus, lack of Mecp 2 may reduce bone density through osteoblastic dysfunction (16).

Other critical factors for optimal bone acquisition are sufficient weight bearing activity and adequate nutrition (15). Physical activity is often limited in RTT with individuals having difficulty in standing and walking, which declines further with age (17). Feeding-related problems are also observed in RTT, with possible consequences for nutritional intake and growth $(18,19)$. Growth retardation is one of the supportive criteria for RTT (20) and may occur independently of nutritional intake.

The main aims of this study were to determine bone mass and density in a national RTT population and to investigate the influence of genotype, epilepsy, BMI, and mobility on these

\footnotetext{
Abbreviations: aBMD, areal bone mineral density; ARSD, Australian Rett Syndrome Database; BMAD, bone mineral apparent density; BMC, bone mineral content; BMD, bone mineral density; DXA, dual energy x-ray absorptiometry; FN, femoral neck; LD, large deletion; CT, late carboxylterminal truncation; LTM, lean tissue mass; LS, lumbar spine; MECP2, methyl-CpG binding protein 2; RTT, Rett syndrome; TICHR, Telethon Institute for Child Health Research; TB, total body
} 
measures. To aid in the interpretation of bone outcomes, the influence of height $Z$ score, bone area (BA), and lean tissue mass (LTM) on BMC at the total body (TB) was also assessed.

\section{METHODS}

The Australian Rett Syndrome Database (ARSD) was established in 1993 and is a population-based register of RTT cases born in 1976 and subsequently (1). Individuals aged $4 \mathrm{y}$ and more were eligible for participation in this study. Ethics approvals were provided by Princess Margaret Hospital in Western Australia and the major Australian hospitals involved. The family of each eligible person was contacted by phone, invited to participate, and informed consent obtained. Appointments were organized at the nearest location for the family with the appropriate DXA equipment.

Densitometry. A scanning protocol was developed by an experienced densitometrist based on standard manufacturer positioning for pediatric and adult patients. Lumbar spine (LS) (L2-L4), left and right femoral neck (FN), and TB scans were obtained. Height and weight were measured immediately before the scan and used to determine the appropriate acquisition mode. The width of the FN region of interest was set at $1.50 \mathrm{~cm}$ for participants with weight $>34 \mathrm{~kg}$ and $1.00 \mathrm{~cm}$ for participants with weight $\leq 34 \mathrm{~kg}$, as this procedure was used by $\mathrm{Lu}$ et al. (21) in the analysis of population controls.

Areal BMD was measured by DXA, using GE-LUNAR Prodigy Densitometers (GE Medical Systems-LUNAR, Madison, WI), and analyzed using proprietary software version 10.50. Outcome measures were aBMD (grams per square centimeters) at the LS and FN; BMC (grams) at the TB; BA (square centimeters); and LTM (grams) (22). To better reflect the volumetric density at the LS, bone mineral apparent density (BMAD) was also calculated $\left(\mathrm{g} / \mathrm{cm}^{3}\right)$ (L1-L4) (22). Scans were performed in seven locations throughout Australia, between August 2005 and February 2008.

All scans were sent electronically to the Telethon Institute for Child Health Research (TICHR) in Perth, Western Australia, in de-identified format. Scans were then reanalyzed in a standardized format, by one trained operator. For patients with spinal rods, only FN scans were included. Scans with movement artifact were also excluded. Normative Australian data for children and adults $(n>800)$ were used to calculate sex-matched height standardized $Z$ scores for aBMD, BMAD, and BMC (21). In our study, an aBMD or BMC height standardized $Z$ score $>1$ SD below zero was classed as low bone density for height and $Z$ scores $>2$ SD below zero was classed as very low bone density for height (23).

Using the "Molgaard approach," the TBBMC was further evaluated (24), including analysis of $Z$ scores for height for age (short or long bones), BA adjusted for height (narrow or wide bones), and TBBMC adjusted for BA. Because of the close relationship between muscle and bone, LTM adjusted for height and TBBMC adjusted for LTM were also investigated (25).

ARSD questionnaires. Questionnaires are completed by caregivers and/or clinicians for all participants on registration into the ARSD. Follow-up questionnaires have been administered every 2 y, since 2000. Data were obtained from the most recently completed questionnaire, on presence of epilepsy, mobility level, fracture history, and pubertal stage. Mobility was classified into three levels, of "walks unaided" (level 1) or "with a degree of unsteadiness" (level 2), "walks with assistance" and "exhibits minimal movement/wheelchair bound" (level 3). Pubertal stage was based on the five Tanner stages of breast development and pubic hair. Mutation data were categorized into 13 groups as follows: each of the eight most common individual MECP2 gene point mutations, large deletions (LD), late carboxylterminal truncation (CT) mutations, and finally the less common $M E C P 2$ gene mutations referred to as the "Other" group (p.R306H, p.S134C, p.P152R, p.P255R, early truncating and exon one). Some participants did not have a $M E C P 2$ mutation elicited or had not been tested. Age was expressed categorically, in four groups $(4-8,>8-14.5,>14.5-20$, and $>20 \mathrm{y})$.

Statistical analysis. Height, weight, and BMI standardized $Z$ scores were calculated using the US Centers for Disease Control and Prevention online data files (26). The Pearson $\chi^{2}$ test and Fisher exact test were used to compare age distribution, epilepsy diagnosis, and mutation type between the study group and the remaining Australian RTT population. The effects of age, epilepsy, BMI, mobility, and mutation type on the TBBMC, LS, and FN aBMD height standardized $Z$ scores were investigated using multivariate linear regression. Tanner stage, previous fracture, and anticonvulsant use were also investigated, using univariate regression models, but found not to be associated with bone outcomes and therefore not included in subsequent models. The mean height standardized $Z$ scores and 95\% CI for the bone outcomes were obtained from the multiple regression models. All statistical analyses were performed using the STATA software version 9 (STATA, College Station, TX).

\section{RESULTS}

In August 2005, there were 274 girls and women with RTT aged $4 \mathrm{y}$ and more living across Australia who were theoretically eligible for this study. However, organizing a DXA scan was only feasible in those who lived within reasonable proximity to one of the major densitometer locations. Nearly half of the group $(n=130)$ had scans arranged by the study team or by their own clinician within the timeframe of the study. Scans in 26 of 130 (20\%) were performed on densitometers other than Lunar and therefore could not be incorporated into the study. Scans were received at the TICHR for 97 of the 104 and thus were available for analysis. For the remaining 144 of the girls and women, arranging a scan was not possible because of lack of contact, geographical location, or difficulty for the child/family.

The mean age of the 97 females on whom a scan was received was 15.0 y ( $\mathrm{SD}=7.1$, range, $4-30.5$ y). Fifty-three participants had all bone outcomes assessed, including the TB, LS, and left and right FN. TBBMC was assessed in 83 participants, and 73 (64 without spinal rods) had LS aBMD measured. The LS BMAD was subsequently calculated for 62 individuals. Scans at the FN each totalled 73.

Comparisons of age, mutation type distribution, and the percentage with epilepsy between the study group and the remaining RTT population are shown in Table 1 . When compared with the remaining RTT population, the mean age of the study group was higher (15.0 versus $10.1 \mathrm{y} ; p=0.001)$ and the proportion of individuals with epilepsy lower $(75 \%$ versus $84 \% ; p=0.063)$. Genetic testing had been performed in $95(98.0 \%)$ of study group participants and the distribution of mutation types was similar to the remaining RTT population $(p=0.363)$. Thus, the distribution of the most common

Table 1. Characteristics of the bone study group and the Australian RTT population older than $4 y$ who did not participate in the study

\begin{tabular}{lcc}
\hline & $\begin{array}{c}\text { Bone study group } \\
(n=97)\end{array}$ & $\begin{array}{c}\text { Remainder of } \\
\text { RTT population } \\
>4 \text { y }(n=177)\end{array}$ \\
\hline Age* & $15.00 \pm 7.16$ & $10.09 \pm 7.10$ \\
Epilepsy† & & \\
No & $24(24.7)$ & $28(16.1)$ \\
Yes & $73(75.3)$ & $146(83.9)$ \\
Mutation† & & $10(5.9)$ \\
C-terminal & $12(12.4)$ & $7(4.1)$ \\
Large deletion & $7(7.2)$ & $37(21.9)$ \\
No mutation & $20(20.6)$ & $20(11.8)$ \\
Other & $9(9.3)$ & $5(3.0)$ \\
p.R106W & $3(3.1)$ & $12(7.16)$ \\
p.R133C & $6(6.2)$ & $9(5.3)$ \\
p.R168X & $10(10.3)$ & $9(5.3)$ \\
p.R255X & $5(5.2)$ & $12(7.1)$ \\
p.R270X & $3(3.1)$ & $11(6.5)$ \\
p.R294X & $7(7.2)$ & $7(4.1)$ \\
p.R306C & $6(6.2)$ & $13(7.7)$ \\
T158M & $7(7.2)$ & $14(8.3)$ \\
Not tested & $2(2.1)$ &
\end{tabular}

Values are expressed as mean $\pm \mathrm{SD}$ or $n(\%)$.

$* p=0.001$.

$\dagger p=0.363$.

$\ddagger p=0.0833$. 
Table 2. Mobility level and epilepsy diagnosis by mutation group

\begin{tabular}{|c|c|c|c|c|c|}
\hline \multirow[b]{3}{*}{ Mutation } & \multicolumn{3}{|c|}{ Mobility level } & \multirow{2}{*}{\multicolumn{2}{|c|}{ Epilepsy }} \\
\hline & \multirow{2}{*}{$\begin{array}{l}\text { Walks } \\
\text { unaided }\end{array}$} & \multirow{2}{*}{$\begin{array}{l}\text { Walks with } \\
\text { assistance }\end{array}$} & \multirow{2}{*}{$\begin{array}{c}\text { Does not walk/ } \\
\text { wheelchair bound }\end{array}$} & & \\
\hline & & & & Yes & No \\
\hline Carboxyl-terminal truncation & $4(33)$ & $3(25)$ & $5(42)$ & $9(75)$ & $3(25)$ \\
\hline Large deletion & $0(0)$ & $1(14)$ & $6(86)$ & $6(86)$ & $1(14)$ \\
\hline No mutation & $3(15)$ & $4(20)$ & $13(65)$ & $18(90)$ & $2(10)$ \\
\hline Other & $1(12)$ & $4(50)$ & $3(38)$ & 7 (78) & $2(22)$ \\
\hline p.R106W & $0(0)$ & $0(0)$ & $3(100)$ & $2(67)$ & $1(33)$ \\
\hline p.R133C & $2(50)$ & $1(25)$ & $1(25)$ & $5(83)$ & $1(17)$ \\
\hline p.R168X & $1(11)$ & $3(33)$ & $5(56)$ & $7(70)$ & $3(30)$ \\
\hline p.R255X & $0(0)$ & $1(20)$ & $4(80)$ & $3(60)$ & $2(40)$ \\
\hline p.R270X & $0(0)$ & $1(33)$ & $2(67)$ & $3(100)$ & $0(0)$ \\
\hline p.R294X & $4(67)$ & $2(33)$ & $0(0)$ & $5(71)$ & $2(29)$ \\
\hline p.R306C & $3(50)$ & $2(33)$ & $1(17)$ & $2(33)$ & $4(67)$ \\
\hline p.T158M & $0(0)$ & $2(40)$ & $3(60)$ & $4(57)$ & $3(43)$ \\
\hline TOTAL & $18(20)$ & $24(27)$ & $46(53)$ & $71(75)$ & $24(25)$ \\
\hline
\end{tabular}

Values are expressed as $n(\%)$.

Table 3. Mean body composition measures (SD) by mutation group

\begin{tabular}{|c|c|c|c|c|c|}
\hline Mutation & Height $Z$ score & BA/height & LTM/height & TBBMC/LTM & TBBMC/BA \\
\hline Carboxyl-terminal truncation & $-0.88(2.58)$ & $-0.58(2.05)$ & $-1.13(1.65)$ & $1.16(1.98)$ & $0.67(1.78)$ \\
\hline Large deletion & $0.64(2.89)$ & $-2.21(1.30)$ & $-1.40(1.15)$ & $-0.40(1.94)$ & $0.69(1.56)$ \\
\hline No mutation & $-1.14(1.80)$ & $-1.28(1.81)$ & $-1.34(1.51)$ & $0.07(1.24)$ & $0.03(1.21)$ \\
\hline Other & $-1.66(2.53)$ & $-0.71(1.53)$ & $-1.28(1.14)$ & $-0.04(1.55)$ & $-1.10(1.01)$ \\
\hline p.R106W & $-1.87(2.05)$ & $-2.89(2.05)$ & $-1.90(1.43)$ & $0.27(0.67)$ & $2.21(1.03)$ \\
\hline p.R133C & $-1.67(3.34)$ & $0.65(1.53)$ & $-0.45(1.18)$ & $2.16(1.44)$ & $0.72(1.19)$ \\
\hline p.R168X & $-2.17(2.40)$ & $-1.78(2.85)$ & $-0.87(2.49)$ & $-0.84(1.85)$ & $0.37(1.29)$ \\
\hline p.R255X & $-0.80(2.26)$ & $-1.91(1.09)$ & $-0.87(0.75)$ & $-0.66(0.79)$ & $1.06(1.74)$ \\
\hline p.R270X & $-3.55(1.41)$ & $-0.06(1.16)$ & $-0.18(0.47)$ & $0.38(0.41)$ & $1.02(3.11)$ \\
\hline p.R294X & $-1.09(1.58)$ & $0.60(1.16)$ & $-0.14(2.25)$ & $0.58(1.40)$ & $-0.30(0.86)$ \\
\hline p.R306C & $1.19(2.73)$ & $-0.33(1.97)$ & $-0.16(1.40)$ & $-0.11(1.26)$ & $0.00(0.50)$ \\
\hline p.T158M & $-1.00(2.38)$ & $-1.94(1.53)$ & $-2.52(1.48)$ & $1.47(3.02)$ & $0.32(1.68)$ \\
\hline TOTAL & $-1.10(2.43)$ & $-1.02(1.97)$ & $-1.10(1.62)$ & $0.32(1.78)$ & $0.28(1.48)$ \\
\hline
\end{tabular}

mutation types in the study group was an adequate representation of that seen within the Australian RTT population.

The mean height and weight were reduced in the study group relative to the general normative population (21), with the height range being 97-167 cm (mean age standardized $Z$ score $=-1.10, \mathrm{SD}=2.42$ ) and the weight range from 11 to $77 \mathrm{~kg}$ (mean age standardized $Z$ score $=-1.24, \mathrm{SD}=3.16$ ). The mean BMI $Z$ score was -1.00 (SD = 2.59). Mobility level and frequency of those with epilepsy in each mutation group are shown in Table 2. Overall, 29 (32.2\%) participants could walk unaided, $15(16.7 \%)$ could walk with assistance, and $46(51.1 \%)$ were wheelchair bound or had minimal movement.

Twenty-six (31.7\%) individuals had previously had one or more fractures. Nineteen $(73.1 \%)$ of the 26 had one or more clinically significant fractures of the lower limb or vertebrae, or two or more long-bone fractures in the upper limb, which are consistent with the International Society for Clinical Densitometry fracture criteria for osteoporosis in children and adolescents (23).

The TBBMC mean height standardized $Z$ score was -0.62 $(\mathrm{SD}=1.52)$. The $\mathrm{LS}$ mean height standardized $Z$ score for aBMD was $-0.72(\mathrm{SD}=2.01)$ and $\mathrm{LS}$ BMAD was -0.60 $(\mathrm{SD}=1.68)$. The mean aBMD height standardized $Z$ scores for the left and right FN were very low, at $-2.15(\mathrm{SD}=1.84)$ and $-2.20(\mathrm{SD}=1.68)$, respectively. Thirty-one $(41.3 \%)$ of the LS aBMD, $37(44.6 \%)$ of the TBBMC, and 57 (78\%) of the right $\mathrm{FN}$ aBMD height standardized $Z$ scores were $>1 \mathrm{SD}$ below the population mean.

Table 3 shows the mean $Z$ score for body composition data for all participants by mutation group. The mean BA adjusted for height was $-1.02(\mathrm{SD}=1.97)$, and mean LTM adjusted for height was $-1.10(\mathrm{SD}=1.61)$. After adjusting for either LTM (mean TBBMC $=0.32, \mathrm{SD}=1.78$ ) or BA (mean $\mathrm{TBBMC}=0.28, \mathrm{SD}=1.48$ ), the mean TBBMC $Z$ score was above zero.

Multivariate linear regression models for each bone outcome (height standardized), included mutation type, age group, epilepsy diagnosis, BMI, and mobility level. Table 4 shows the mean height standardized $Z$ score and CI obtained from the regression models for the LSaBMD, right FN, and TBBMC by age group, epilepsy diagnosis, and mobility. There were contrasting results between the effects of age on the LS aBMD and FN. When compared with the 4-8 y old group, the LS aBMD was higher in individuals in age groups $>14.5-20$ and $>20$ y (mean $Z$ scores $>14-20=0.46$ and 1.11 , respectively; $p=0.009$ ), whereas the FN aBMD was substantially lower in those older than $20 \mathrm{y}$ (mean $Z$ score $=$ -3.07 ) compared with the younger age groups (mean $Z$ scores $>14-20=-1.31,>8-14.5=-1.63,4-8=-1.00 ; p=$ 
Table 4. Mean height standardized Z score and CI for each bone outcome by age group, epilepsy diagnosis, and mobility level

\begin{tabular}{|c|c|c|c|}
\hline & LS aBMD & Right FN aBMD & TBBMC \\
\hline \multicolumn{4}{|l|}{ Age group (y) } \\
\hline $4-8$ & $-1.40(-3.14,0.33)$ & $-1.00(-3.09,1.10)$ & $0.26(-1.10,1.62)$ \\
\hline$>8-14.5$ & $-1.21(-2.73,0.31)$ & $-1.63(-3.23,-0.04)$ & $-0.39(-1.57,0.79)$ \\
\hline$>14.5-20$ & $0.46(-1.24,2.15)$ & $-1.31(-3.11,0.47)$ & $0.19(-1.10,1.49)$ \\
\hline$>20-31$ & $1.11(-0.84,3.06)$ & $-3.07(-5.02,-1.12)$ & $0.08(-1.27,1.44)$ \\
\hline \multicolumn{4}{|l|}{ Epilepsy } \\
\hline No & $-1.40(-3.14,0.33)$ & $-1.00(-3.09,1.10)$ & $0.26(-1.10,1.62)$ \\
\hline Yes & $-1.73(-3.37,-0.09)$ & $-2.32(-2.64,-2.00)$ & $-0.01(-1.31,1.30)$ \\
\hline \multicolumn{4}{|l|}{ Mobility } \\
\hline Unaided & $-0.30(-1.75,1.14)$ & $0.01(-1.98,2.00)$ & $0.63(-0.61,1.87)$ \\
\hline Assistance & $-0.59(-2.11,0.92)$ & $-1.95(-3.07,0.81)$ & $-1.71(-0.28,2.04)$ \\
\hline Wheelchair & $-1.40(-3.14,0.33)$ & $-1.00(-3.09,1.10)$ & $0.26(-1.10,1.62)$ \\
\hline
\end{tabular}

The reference groups used in the models were the "CT" mutation group, the "walks unaided" mobility group, and the "no epilepsy" group.

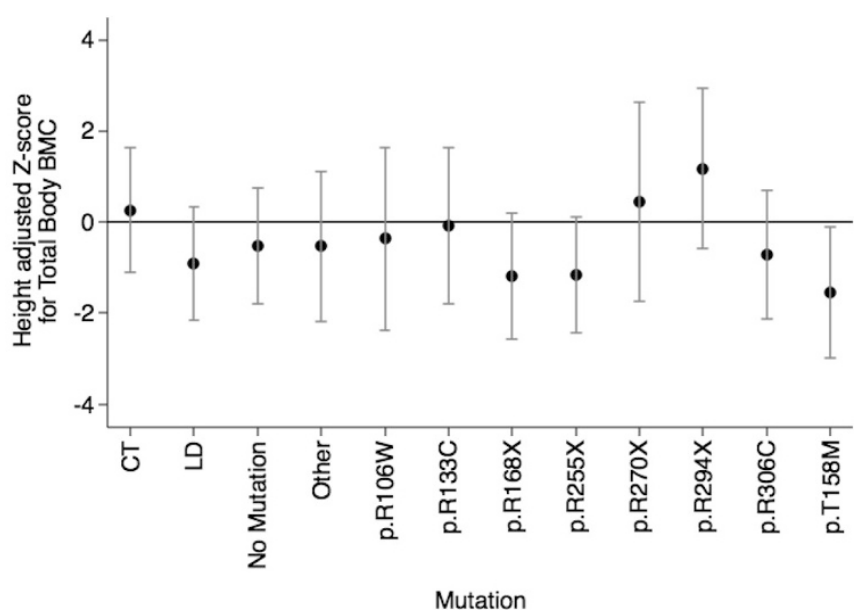

Figure 1. Mean height standardized $Z$ score and CI for TBBMC in each mutation group.

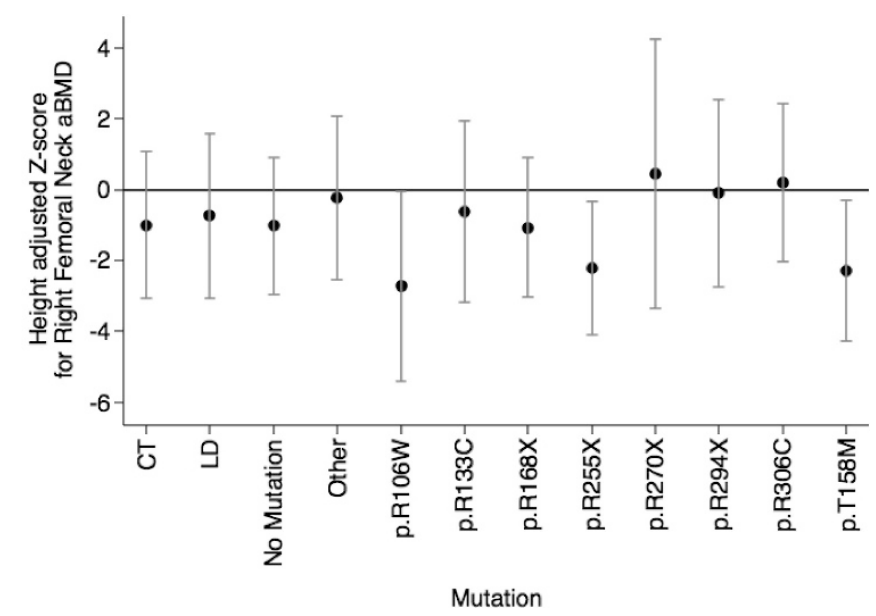

Figure 2. Mean height standardized $Z$ score and CI for LS aBMD in each mutation group.

0.026). Although the mean height standardized $Z$ scores for the three bone outcomes were lower for those with epilepsy, only the FN $Z$ scores were significantly different (mean $Z$ scores $=-1.00$ without and -2.32 with epilepsy; $p=0.005$ ). The aBMD and BMC mean height standardized $Z$ scores were lower in the "walks with assistance" and "exhibits minimal movement/wheelchair bound" mobility groups.

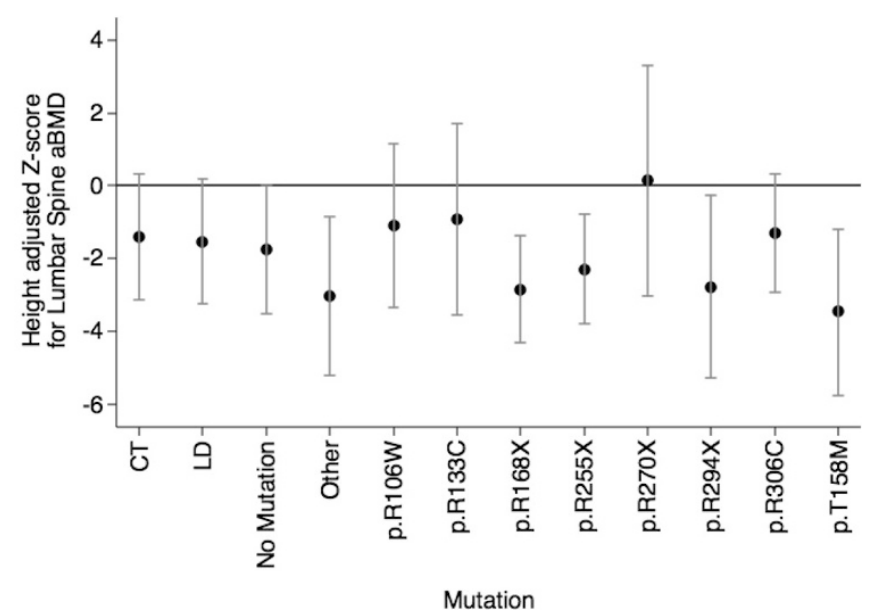

Figure 3. Mean height standardized $Z$ score and CI for right femoral neck aBMD in each mutation group.

Figures 1-3 show the adjusted mean height standardized $Z$ scores and CI for the TBBMC, LSaBMD, and right FN by mutation group, from the multivariate regression models. The p.R168 $\times($ mean $=-1.20)$ and p.T158M $($ mean $=-1.54)$ mutation groups had significantly lower height adjusted TBBMC than the CT reference group (mean $=0.26$ ). The negative impact of the presence of these mutations can also be seen for LS aBMD height standardized $Z$ score (p.R168 $\times$ mean $=-2.85$, p.T158M mean $=-3.47, \mathrm{CT}$ mean $=$ -1.40 ). The mean height standardized $Z$ score for both the p.R168 $\times$ and p.T158M mutation groups was also low at the FN but not appreciably different to the CT group.

\section{DISCUSSION}

This study showed that individuals with RTT have low aBMD and BMC, particularly at the FN. BA was also reduced for height. The mean aBMD height standardized $Z$ score at the FN decreased with age and presence of epilepsy. Those with less mobility also had lower mean values for all bone outcomes. Regression models showed that the strongest predictors for low aBMD and BMC were the p.R168 $\times$ and p.T158M mutation types. Body composition analysis showed that height, weight, BMI, and LTM adjusted for height were lower than that expected for age. However, when TBBMC was 
adjusted for either LTM or BA, results approximated the population norm.

A major strength of the study was that the participants were a close representation of the population-based Australian RTT cohort, thus supporting the generalization of findings to all individuals with RTT. Comprehensive data were available on each participant, increasing the number of factors that could be included in the analysis. A detailed universal scanning protocol was developed and adopted by all centers. All scans were reanalyzed by the same operator, thus maintaining consistency in highlighting regions of interest. Internal calibrations were performed within each scan location and each center used the same type of densitometer. Results were calibrated against Australian normative data.

To accommodate the decreased stature seen in RTT $(18,19)$, calculation of height standardized $Z$ scores and BMAD were made. Using the "Molgaard approach" (24) and another similar (25) approach, a more in depth interpretation of TBBMC was made, by adjusting the bone outcome for LTM and BA. Thus, we examined bone mineralization taking into account the effects of short stature, narrow bones and reduced muscle mass.

Although DXA is the commonly used method for evaluating pediatric bone mass and density, its bone outcomes are strongly influenced by bone size and are thus highly growth dependent (22). LTM, as measured by DXA, is a major predictor of BMC and accords with other methods of measuring muscle mass (25). As muscle action delivers the largest loads and bone strains (27), participants with low muscle mass for height should have a proportionately low BMC.

Performing this analysis on the study group, with a mean TBBMC height standardized $Z$ score of -0.62 , bones were short (as indicated by a low mean height $Z$ score of -1.10 ), narrow (low mean BA adjusted for height $Z$ score of -1.02), but with normal mineralization for bone area (normal mean TBBMC adjusted for BA of 0.28). Notably, there was low LTM for height (mean $Z$ score $=-1.10$ ) indicating low muscle mass; however, when bone mineralization was adjusted for muscle mass, the result was normal (TBBMC adjusted for LTM of 0.32). These findings indicate only a slightly reduced bone mineralization, due largely to low BA (narrow bones) and low muscle mass (low muscle pull) on slightly short bones. It is interesting that the mutations associated with the lowest height adjusted BMC/aBMD (at all sites) had the lowest bone length $(\mathrm{R} 168 \times$ height $Z$ score $=$ -2.17 ), narrowest bones (BA height adjusted $\mathrm{z}$ scores -1.78 and -1.94 for $\mathrm{R} 168 \times$ and $\mathrm{T} 158 \mathrm{M}$, respectively), and lowest muscle mass (LTM/ height adjusted $Z$ score $=-2.52$ for T158M). It is illustrative that individuals with these two mutations, with the greatest clinical severity, including lower mobility and a high prevalence of epilepsy (28), have the most severe bone mineral phenotype.

Downs et al. (6) previously found fracture rate in this RTT population to be four times that of the general population and those with the p.R $168 \times$ and p.R $270 \times$ mutations at particularly increased risk. Unexpectedly in this study, which unlike the fracture study is by its nature limited to live cases, the p.R270 $\times$ mutation was not a strong predictor of bone outcome measures. The three participants with this mutation, aged between 18 and $30 \mathrm{y}$, may represent a survivor bias and may be functionally more able than typically seen with this mutation (28). Downs et al. (6) found the most common fracture site to be the femur, which correlates with the findings in this study, where the FN region (and the legs, data not shown) had the lowest aBMD values. While the difficulties associated with FN positioning are acknowledged, a likely clinical explanation for the very low FN measurements may be that more than half of the participants were predominantly wheelchair bound. However, as scans for participants with spinal rods could only be analyzed at the $\mathrm{FN}, \mathrm{FN}$ results may be more representative of the total RTT population, with LS and TB results biased toward those with less severe phenotypes, with less scoliosis and more mobility.

The reduced height and BA for age found in this study suggests that girls with RTT have bones both shorter and more narrow than expected. Reduced height, weight, and BMI have already been well documented in RTT $(18,19)$. Reassuringly, evidence of a low calcium intake in females with RTT has not been identified, either in an Australian $(n=84)$ (11) or US study $(n=10)$ (29). However, another study did identify reduced serum 25 hydroxyvitamin D levels (12). It may well be beneficial to monitor vitamin D levels in RTT and supplement as necessary. On the other hand, biochemical analysis of serum calcium, phosphate, alkaline phosphatase, and PTH levels in RTT were not shown to differ from controls in a sample of 82 individuals (12). These normal biochemical findings suggest that vitamin D deficiency, if present, is not severe.

However, the major explanation for the reduced BA and BMD observed in this study is the decreased LTM. The major physiological loads placed on bone originate from the pull of muscles, as they contract (30). This in turn causes remodelling of the bone to alter (in particular) the bone geometry, thus increasing the strength of bone, such that it is appropriate to the loads placed upon it (30). As LTM largely represents skeletal muscle mass, a surrogate of muscle action, any reduction in LTM will influence bone size and geometry (31). Reduced LTM in this cohort was not surprising, as the majority $(80 \%)$ of participants were unable to walk independently or were wheelchair bound. LTM has been found to be a strong predictor of BMD and BMC in adults and in normally developing children and adolescents $(25,31)$. The dual findings in this study, that TBBMC was normalized after adjustment for BA and LTM and that those who were less mobile had lower aBMD and BMC values together, support the contention that mobility is a major factor in the osteopenia of RTT.

In this study, aBMD at the FN decreased with age and all bone outcomes were lower in those diagnosed with epilepsy. Our findings are in agreement with a recent US study performed in 50 females with RTT, aged between 2 and $38 \mathrm{y}$ (14). This US study also found that previous anticonvulsant use, previous fractures, and the presence of scoliosis all had negative associations with LS, FN, and TB bone measurements (14). In our study, these factors were not significant predictors of aBMD or BMC. On the other hand, the US study 
was unable to demonstrate any relationship between genotype and bone density, potentially because of the small sample size, which only allowed mutation categorization into five broad groups (14). Our study included access to a larger cohort of individuals, allowing more refined categorisation of mutations. Furthermore, our study also investigated the impact of BA and LTM on TBBMC measurements.

Necessary limitations to our study included reduction in the sample size by exclusion of scans where spinal rods were present or from movement artifact. Furthermore, height may have been underestimated in those (more severely affected) individuals with scoliosis, leading to an overestimation of their aBMD or BMC height standardized $Z$ scores.

In conclusion, individuals with RTT have low aBMD, BMC, BA, and LTM measures, which is only partially accounted for by their decreased height. Bone outcome measures were particularly low at the FN, the most common site of fracture, and decreased at this site with age. Those with the more severe p.R168 $\times$ and p.T158M mutations were at greatest risk of low bone density. Wheelchair bound individuals and those with epilepsy had lower bone outcome measures. Although we identified a strong relationship between genotype and BMC and aBMD, there was also a clear relationship between LTM, mobility, and bone outcome measures. Thus, maintaining mobility, particularly in those with high-risk mutations, has potential as an environmental modification to optimize bone health. In future research, use of a 3D technology, such as high resolution Peripheral Quantitative Computed Tomography, could give additional insights into compartmental mineralization, microstructure, and geometry and their interaction with muscle. It is our recommendation that guidelines for the management of bone health in individuals with RTT should be developed and implemented, early in life.

Acknowledgments. We thank Carol Philippe from the Telethon Institute for Child Health Research (Perth) who was responsible for organizing the bone density appointments; Christine Kennelly at Perth Radiology Clinic (Western Australia), Anne Glue at Benson Radiology (Adelaide), staff at the Body Composition Laboratory, Monash Medical Centre (Victoria), and staff from the Department of Nuclear Medicine at the Royal Brisbane \& Women's Hospital (Queensland) for their contributions to the study; and Medtel Australia (Perth) for providing the study with the GE Healthcare Encore 2006 software. We express our sincere gratitude to all the families who have contributed to the study by completing questionnaires and arranging for their daughters to have scans, and the Australian Pediatric Surveillance Unit and the Rett Syndrome Association of Australia for facilitating case ascertainment in Australia.

\section{REFERENCES}

1. Laurvick CL, de Klerk N, Bower C, Christodoulou J, Ravine D, Ellaway C, Williamson S, Leonard H 2006 Rett syndrome in Australia: a review of the epidemiology. J Pediatr 148:347-352
2. Hagberg BA, Skjeldal OH 1994 Rett variants: a suggested model for inclusion criteria. Pediatr Neurol 11:5-11

3. Colvin L, Leonard H, de Klerk N, Davis M, Weaving L, Williamson S, Christodoulou J 2004 Refining the phenotype of common mutations in Rett syndrome. J Med Genet 41:25-30

4. Renieri A, Meloni I, Longo I, Ariani F, Mari F, Pescucci C, Cambi F 2003 Rett syndrome: the complex nature of a monogenic disease. J Mol Med 81:346-354

5. Christodoulou J, Weaving LS 2003 MECP2 and beyond: phenotype-genotype correlations in Rett syndrome. J Child Neurol 18:669-674

6. Downs J, Bebbington A, Woodhead H, Jacoby P, Jian L, Jefferson A, Leonard H 2008 Early determinants of fractures in Rett syndrome. Pediatrics 121:540-546

7. Leonard H, Downs J, Jian L, Bebbington A, Jacoby P, Nagarajan L, Ravine D, Woodhead H 2010 Valproate and risk of fracture in Rett syndrome. Arch Dis Child 95:444-448

8. Hui SL, Slemenda CW, Johnston CC Jr 1988 Age and bone mass as predictors of fracture in a prospective study. J Clin Invest 81:1804-1809

9. Flynn J, Foley S, Jones G 2007 Can BMD assessed by DXA at age 8 predict fracture risk in boys and girls during puberty?: an eight-year prospective study. J Bone Miner Res 22:1463-1467

10. Leonard H, Thomson R, Bower C, Fyfe S, Constantinou J 1995 Skeletal abnormalities in Rett syndrome: increasing evidence for dysmorphogenetic defects. Am J Med Genet 58:282-285

11. Leonard H, Thomson M, Glasson E, Fyfe S, Leonard S, Bower C, Christodoulou J, Ellaway C 1999 A population based approach to the investigation of osteopenia in Rett syndrome. Dev Med Child Neurol 41:323-328

12. Cepollaro C, Gonnelli S, Bruni D, Pacini S, Martini S, Franci MB, Gennari L, Rossi S, Hayek G, Zappella M, Gennari C 2001 Dual X-ray absorptiometry and bone ultrasonography in patients with Rett syndrome. Calcif Tissue Int 69:259-262

13. Haas RH, Dixon SD, Sartoris DJ, Hennessy MJ 1997 Osteopenia in Rett syndrome. J Pediatr 131:771-774

14. Motil KJ, Ellis KJ, Barrish JO, Caeg E, Glaze DG 2008 Bone mineral content and bone mineral density are lower in older than younger females with Rett syndrome. Pediatr Res 64:435-439

15. Davies JH, Evans BA, Gregory JW 2005 Bone mass acquisition in healthy children. Arch Dis Child 90:373-378

16. O'Connor RD, Zayzafoon M, Farach-Carson M, Schanen N 2009 Mecp2 deficiency decreases bone formation and reduces bone volume in a rodent model of Rett syndrome. Bone 45:346-356

17. Downs JA, Bebbington A, Jacoby P, Msall ME, McIlroy O, Fyfe S, Bahi-Buisson N, Kaufmann WE, Leonard H 2008 Gross motor profile in Rett syndrome as determined by video analysis. Neuropediatrics 39:205-210

18. Oddy WH, Webb KG, Baikie G, Thompson SM, Reilly S, Fyfe SD, Young D, Anderson AM, Leonard H 2007 Feeding experiences and growth status in a Rett syndrome population. J Pediatr Gastroenterol Nutr 45:582-590

19. Reilly S, Cass H 2001 Growth and nutrition in Rett syndrome. Disabil Rehabil 23:118-128

20. Hagberg B, Hanefeld F, Percy A, Skjeldal O 2002 An update on clinically applicable diagnostic criteria in Rett syndrome. Comments to Rett Syndrome Clinical Criteria Consensus Panel Satellite to European Paediatric Neurology Society Meeting, Baden Baden, Germany, 11 September 2001. Eur J Paediatr Neurol 6:293-297

21. Lu PW, Briody JN, Ogle GD, Morley K, Humphries IR, Allen J, Howman-Giles R, Sillence D, Cowell CT 1994 Bone mineral density of total body, spine, and femoral neck in children and young adults: a cross-sectional and longitudinal study. J Bone Miner Res 9:1451-1458

22. Fewtrell MS, Group BP 2003 Bone densitometry in children assessed by dual x ray absorptiometry: uses and pitfalls. Arch Dis Child 88:795-798

23. Baim S, Leonard MB, Bianchi M-L, Hans DB, Kalkwarf HJ, Langman CB, Rauch F 2008 Official Positions of the International Society for Clinical Densitometry and executive summary of the 2007 ISCD Pediatric Position Development Conference. J Clin Densitom 11:6-21

24. Mølgaard C, Thomsen BL, Prentice A, Cole TJ, Michaelsen KF 1997 Whole body bone mineral content in healthy children and adolescents. Arch Dis Child 76:9-15

25. Högler W, Briody J, Woodhead HJ, Chan A, Cowell CT 2003 Importance of lean mass in the interpretation of total body densitometry in children and adolescents. J Pediatr 143:81-88

26. Centers for Disease Control National health and nutrition examination survey: CDC growth charts. 2002. Available at: http://www.cdc.gov/growthcharts. Accessed October 8,2008

27. Frost HM, Schnau E 2000 The "muscle-bone unit" in children and adolescents: a 2000 overview. J Pediatr Endocrinol Metab 13:571-590

28. Jian L, Archer H, Ravine D, Kerr A, de Klerk N, Christodoulou J, Bailey ME, Laurvick C, Leonard H 2005 p.R270X MECP2 mutation and mortality in Rett syndrome. Eur J Hum Genet 13:1235-1238

29. Motil KJ, Schultz RJ, Abrams S, Ellis KJ, Glaze DG 2006 Fractional calcium absorption is increased in girls with Rett syndrome. J Pediatr Gastroenterol Nutr 42:419-426

30. Janz KF, Gilmore JM, Burns TL, Levy SM, Torner JC, Willing MC, Marshall TA 2006 Physical activity augments bone mineral accrual in young children: the Iowa Bone Development study. J Pediatr 148:793-799

31. Wang MC, Bachrach LK, Van Loan M, Hudes M, Flegal KM, Crawford PB 2005 The relative contributions of lean tissue mass and fat mass to bone density in young women. Bone 37:474-481 